\title{
Direct Algebraic Reconstruction and Optimal Sampling in Vector Field Tomography
}

\author{
Laurent Desbat and Andreas Wernsdörfer
}

\begin{abstract}
Vector field tomography has been proven to be a very powerful technique for the noninvasive determination of vector field distribution such as in the case of a fluid velocity field. We show that classical tomographic sampling conditions can essentially be applied to vector field tomography. Thus, essentially the same sampling schemes are obtained, and the interlaced scheme is also shown to be the most efficient scheme in vector field tomography. We then propose a Direct Algebraic approach for vector field tomography, with an efficient and robust algorithm for interlaced schemes. Numerical experiments showing the superiority of interlaced schemes are provided.
\end{abstract}

\section{INTRODUCTION}

B $Y$ developing vector field tomography in recent years, a very powerful device has become available for determining the distribution of a vectorial quantity from noninvasive measurement. The tomographic reconstruction of fluid velocity fields was proposed in [2], [15], [20] as an important application. Theses articles describe the reconstruction problem using one of two different reconstruction methods. In the first type of method the problem of reconstructing the vector field is led back to reconstructing a scalar field from which the vector field can be calculated. In the second, using algebraic methods, the vector components are directly computed.

In the first type of approach, the decomposition of vector fields into two parts is utilized, according to Helmholtz's theorem:

$$
\vec{v}=\vec{v}_{w}+\vec{v}_{q} .
$$

$\vec{v}_{w}$ denotes the source-free (solenoidal) and $\vec{v}_{q}$ the curl-free (irrotational) part of the field. In the case of a 2-D field in a plane, each part is then described by a scalar field. The sourcefree components $\vec{v}_{w}$ can be expressed by the vector potential $w \vec{e}_{z}$ and the curl-free components $\vec{v}_{q}$ by the scalar potential $q$ :

$$
\begin{gathered}
\vec{v}_{w}=\nabla \times w \vec{e}_{z}=\left[\frac{\partial w}{\partial x_{2}},-\frac{\partial w}{\partial x_{1}}\right]^{T} \\
\vec{v}_{q}=\nabla q=\left[\frac{\partial q}{\partial x_{1}}, \frac{\partial q}{\partial x_{2}}\right]^{T}
\end{gathered}
$$

Manuscript received March 8, 1994; revised January 20, 1995. The associate editor coordinating the review of this paper and approving it for publication was Prof. Stanley J. Reeves.

L. Desbat is with the laboratory of Image Processing, Modeling and Cognition Techniques, TIMC-IMAG, URA CNRS 1618 Faculté de Médecine, Grenoble, France

A. Wernsdörfer is with the Institute for Measure and Regulation, IMR, Karlsruhe University, Karlsnuhe, Germany.

IEEE Log Number 9412610

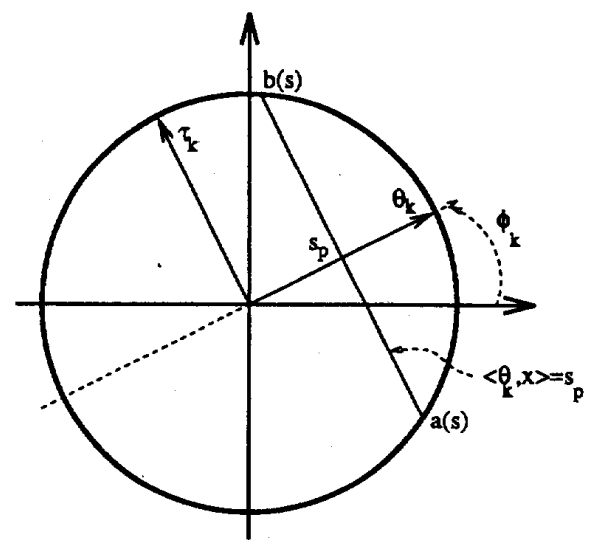

Fig. 1. Geometry parameters of the Radon transform.

Here, $\vec{e}_{z}$ denotes the unit vector normal to the investigated plane. In [2], it is shown that for vector tomography two types of interaction between the vector field and the ray used must be distinguished. The first, which is called the longitudinal interaction, is mathematically expressed by

$$
y_{l}(\phi, s)=\int_{\langle x, \theta\rangle=s}\langle\vec{v}(x), \tau\rangle_{\mathbb{R}^{2}} d x
$$

$\tau=(-\sin \phi, \cos \phi)^{t}$ denotes the unit vector in the direction of the beam propagation. $\theta=(\cos \phi, \sin \phi)^{t}$ is the unit vector normal to the ray in the investigated plane such that $\tau$ is the unit vector obtained from $\theta$ by a rotation of $\pi / 2$; see Fig. 1. $\langle f, g\rangle_{H}$ denotes the inner product of the Hilbert space $H$ (in (3), the Euclidean product in $\mathbb{R}^{2}$ ). Only the components following the direction of the beam are determined by $y_{l}$. We reserve the notation $\vec{f}$ for vectorial functions to underline the difference with scalar tomography; other classical vectorial quantities, such as $\theta, \tau$, are denoted as usual. If this effect is used, only the source-free component $\vec{v}_{w}$ can be measured, and the curl-free part $\vec{v}_{q}$ is lost. The second measuring effect, which is called the transversal interaction, determines the field perpendicular to the beam, and only the curl-free part is measured:

$$
y_{t}(\phi, s)=\int_{\langle x, \theta\rangle=s}\langle\vec{v}(x), \theta\rangle_{\mathbb{R}^{2}} d x .
$$

In both cases, $\Omega$, which is the support of $\vec{v}$, is supposed to be compact. Reconstruction formulas of $\vec{v}$ from (3) and (4) have 
been proposed [2]. However, in most of the practical applications, only $y_{l}$ is measured, and thus, only $v_{w}$ is reconstructed from the tomographic data. In the following, we consider only this problem.

One of the most important questions in such inverse problems in which a function has to be computed from measurements is the efficiency of the measurement geometry. The Fourier analysis approach introduced in [19], developed in [13], and generalized in [8] and [5] leads to sampling conditions and the optimal interlaced scheme in scalar tomography. Let us remark that the interlaced scheme was first proposed by Cormack [4] using elegant geometric arguments. The approach developed in [13] can be used in vector tomography for establishing sampling conditions and the optimality of the interlaced scheme. Recent developments on Fourier theory [9], on sampling conditions, and on efficient schemes for the fan-beam data [14] could also be applied in vector tomography.

Using (3), only the solenoidal component can be reconstructed from the data, and the problem of reconstructing the vector field leads to the problem of calculating the scalar field $w$. This field can be computed by the filtered-backprojection or the direct-fourier method, which have been previously extended and changed for processing the data measured over the vector fields. The solenoidal part is then calculated from the derivatives (2). If $y_{t}(\phi, s)$ is measurable, then the same operation can be applied to (4), and the irrational component can also be estimated. Unfortunately, in many practical applications, only $y_{l}(\phi, s)$ is measured. In this case, if the field $\vec{v}$ is divergenceless, the value of its normal component on the boundary $\partial \Omega$ allows us to compute $q$ (see [16]), and thus, by using the tomographic data $y_{l}$, the vector field $\vec{v}$ can be completely determined.

In [20] and [21], an algebraic approach is proposed, which directly yields the vector components. Here, the investigated plane is discretized by a grid of pixels, and the line integral (3) or (4) is approximated by a weighted sum over the vector components inside those pixels intersected by the ray. The measurement leads to a set of linear equations. Hence, the problem of reconstruction is converted to the problem of solving this set. The system matrix is, in general, not square and is ill conditioned. The solution is computed iteratively [21] or by a singular value decomposition [20], in order to obtain a normal solution.

The filtered-backprojection algorithm is the most popular and probably the most efficient reconstruction method for a large amount of data. It has been adapted to interlaced sampling. This algorithm works perfectly if sufficient care is taken for the backprojection algorithm in the interpolation step (see [8] and references therein). However, this algorithm is based on Radon transform inversion formulas since the first one was originally given by Radon [18]. When reconstructing from very few data (say, 100), these methods cannot be easily justified. Is Radon transform still usable then in this case? In fact, algebraic methods can be more easily justified and seem to provide better reconstructions.

In contrast, for the algebraic methods, not only the measured data are assumed to be discrete, but the object is as well. The discrete pixel model used in ART is natural in order to visualize the solution but quite arbitrary from a mathematical point of view.

Both Buonocore [3] and Natterer [12] proposed a direct algebraic (DA) method for scalar field reconstructions. This method takes into account the discrete nature of the measured data and simply supposes that the investigated object lies in a Sobolev space (the square integrable function space $L^{2}(\Omega)$ is generally considered). Regularization methods have also been proposed for this approach in [10]. In our work, this model is extended for vector tomography. It is shown that the numerically pleasant features are preserved so that an equally efficient algorithm can be used just as in the scalar case.

In Section II, we present an adaptation of the well-known efficient sampling scheme in vector tomography. The vector field tomography sampling requirements are shown to be essentially the same as those in scalar tomography. In Section III, we present the DA approach for vector tomography. An algorithm for efficient geometry is proposed. An efficient implementation of this algorithm is proposed in Section IV. In Section V, we present numerical experiments. They show that the interlaced scheme provides almost as good a reconstruction as the standard scheme from half as many samples as the standard scheme.

\section{EFFICIENT SAMPLING}

\section{A. Introduction}

To save both time and money, it is of great interest to reduce the number of data to a minimum. The Radon transform of the investigated object is sampled by measurement and, hence, as for any other sampling problem, this minimum is given by a sampling theorem. For scalar tomography, this was originally formulated by Rattey and Lindgren [19]. They have also shown that for the scalar case, the band region of a Radon transform (which is decisive for the necessary amount of sampling points) has a very specific shape, which is called the bowtie shape, which is denoted by $\mathbf{S}$ in the following. The Natterer's mathematical approach [13] gives a precise definition of $\mathbf{S}=\{(m, \sigma) ;|\sigma|<b,|m|<\max (|\sigma| / \vartheta,(1 / \vartheta-1) b\}$, where $m, \sigma$ are the respective variables of the suitable Fourier transform of $R f(\phi, s)$ (see [8], [9], and [13] for precise definitions and generalizations), $b$ is essentially the bandwidth of the measured function $f$, and $0<\vartheta<1$ (real arbitrary close to 1 introduced for mathematical reasons). The Petersen and Middleton sampling theorem [17] allows sampling of a function on lattices, i.e., sets of the form $\left\{W k, k \in \mathbb{Z}^{2}\right\}$, where $W$ is a nonsingular matrix, and the sampling points are the whole linear combinations of the column vectors of matrix $W$. The generalized nonoverlapping condition (Nyquist-Shannon) means simply that the sets $\mathbf{S}+2 \pi W^{-t} k$, with $k \in \mathbb{Z}^{2}$ are disjoint (where $S$ is the support of the Fourier transform of the function to be sampled). If a rectangular sampling grid of the Radon transform is assumed, with respect to the sampling theorem, the repetitions of the bowties are placed side by side. As is shown in Fig. 2 (left), the frequency plane is not efficiently utilized in this case. Due to the specific shape of $\mathbf{S}$, there is a lot of free space between adjacent spectra. If other 


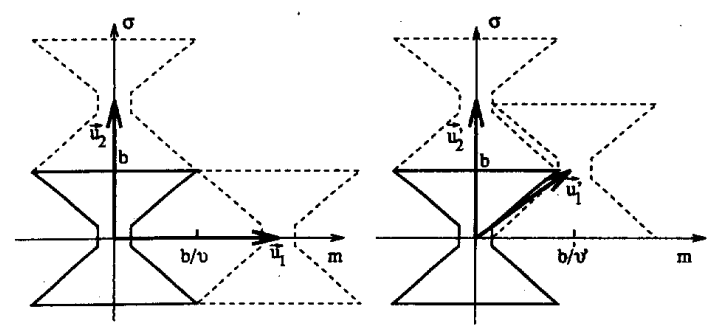

Fig. 2. Sets $\mathbf{S}$ (bold line) and translations (dashed line) on the reciprocal lattice of the sampling scheme, i.e., $\mathbf{S}+2 \pi W_{S}^{-t} k$ (left, $W_{S}$ is the standard sampling matrix) and $\mathbf{S}+2 \pi W_{I}^{-t} k$ (right, $W_{I}$ is the interlaced sampling matrix). Sampling the Radon transform with a rectangular scheme leads to a lattice in the Fourier space generated by $\vec{u}_{1}, \vec{u}_{2}$ (see left) for satisfying the nonoverlapping condition (generalized Nyquist-Shannon condition). The lattice generated by the nonorthogonal vectors $\vec{u}_{1}^{\prime}, \vec{u}_{2}$ (right) satisfies the nonoverlapping condition more efficiently. It corresponds to the interlaced scheme.

sampling schemes are chosen, the bowties can be packed in a more efficient way. In Fig. 2 (right), such an alternative scheme with the corresponding distribution of the bowties in the frequency plane is shown. Although only half of the necessary sampling points in direction of the radial coordinate $s$ are used and, therefore, half of the amount of measured data is saved, the bowties do not overlap, and no aliasing occurs.

As a precondition of such efficient 2-D sampling grids, an exact knowledge is needed of the support of the Fourier transform of the sampled function. In order to also use such schemes for vector field tomography, the support of the Fourier transform of the vector field projection is investigated below. A very intuitive approach shows that interlaced sampling is efficient in vector field tomography and allows sparing of half of the measurements compared to the standard approach. Moreover, the sampling conditions are essentially the same as in scalar tomography. A approach based on Natterer's mathematical proof [13] will be given elsewhere.

First, however, we want to be precise about extensions of classical definitions (see [13]) used in the next section. We will use the following definitions of the 1-D and 2-D Fourier transforms:

$$
\begin{aligned}
\hat{f}(\sigma) & =\frac{1}{\sqrt{2 \pi}} \int_{\mathbb{R}} e^{-i s \sigma} f(\dot{s}) d s, \text { and } \hat{f}(\xi) \\
& =\frac{1}{2 \pi} \int_{\mathbb{R}^{2}} e^{-i\langle x, \xi\rangle_{\mathbb{R}^{2}}} f(x) d x .
\end{aligned}
$$

For a vector field $\vec{v}=\left(v_{1}, v_{2}\right)$, we will denote $\tilde{\vec{v}}=\left(\hat{v}_{1}, \hat{v}_{2}\right)$. Let $y_{\theta}(s)=y(\phi, s)$ be a function defined by (3) or (4). We will use the 1-D Fourier transform of $y_{\theta}(s)\left(=y_{\theta(\phi)}(s)\right)$ :

$$
\hat{y}(\phi, \sigma)=\hat{y_{\theta}}(\sigma)=\frac{1}{\sqrt{2 \pi}} \int_{\mathbb{R}} e^{-i s \sigma} y_{\theta}(s) d s .
$$

We will also make use of the Fourier transform of periodic functions:

$$
\hat{y}_{m}(\sigma)=\frac{1}{2 \pi} \int_{0}^{2 \pi} e^{-i m \phi} \hat{y}(\phi, \sigma) d \phi, \text { where } m \in \mathbf{Z} .
$$

Note that for simplicity, $\hat{y}_{m}(\sigma)$ will sometimes be written $\hat{y}(m, \sigma)$, particularly in Section II-B.

\section{B. Interlaced Sampling for Vector Field Tomography}

Usually, only the longitudinal effect is measured, and (3) can be rewritten as

$$
\begin{aligned}
y_{l} & =\int_{a(s)}^{b(s)} v_{2} \cos \phi-v_{1} \sin \phi d r \\
& =\cos \phi \int_{a(s)}^{b(s)} v_{2} d r-\sin \phi \int_{a(s)}^{b(s)} v_{1} d r \\
& =\cos \phi y_{2}-\sin \phi y_{1}
\end{aligned}
$$

where $a(s)$ and $b(s)$ describe the aperture function of the domain $\Omega$. For the present, the projection of a vector field can be interpreted as a superposition of the amplitude-modulated Radon transform of two scalar fields $v_{1}$ and $v_{2}$. In the frequency domain, the amplitude-modulation causes a widening of the support of $\hat{y}_{2}(m, \sigma)$ and $\hat{y}_{1}(m, \sigma)$ by $\Delta m=1$ and, therefore, a widening of the support of $\hat{y}_{l}(m, \sigma)$ :

$$
\begin{gathered}
\hat{y}_{l}(m, \sigma)=1 / 2\left[\hat{y}_{2}(m+1, \sigma)+\hat{y}_{2}(m-1, \sigma)\right]- \\
(i / 2)\left[\hat{y}_{1}(m+1, \sigma)-\hat{y}_{1}(m-1, \sigma)\right] .
\end{gathered}
$$

For the definition of the bandwidth of $\hat{y}_{l}(m, \sigma)$ in the direction of $\sigma$, the largest of the two bandwidths of the scalar functions $b_{1}$ or $b_{2}$ is chosen $\left(b=\max \left(b_{1}, b_{2}\right)\right)$. Equation (6) already clearly shows that efficient interlaced sampling exists with apparently only a few more points than in the scalar case (the Fourier support is apparently just a bit larger).

\section{Sampling Schemes}

It can be stated that the support of the Fourier transform of a vector field projection has the same bowtie-shape support as in the scalar case. Thus, the application of efficient 2-D sampling schemes is also possible for vector field tomography.

In scalar tomography (see [13]), we use the well-known standard (points $\left.W_{S}\left(k_{1}, k_{2}\right)^{t},\left(k_{1}, k_{2}\right) \in \mathbb{Z}^{2}\right)$ and interlaced (points $\left.W_{I}\left(k_{1}, k_{2}\right)^{t}\right)$ schemes with

$$
W_{S}=\left(\begin{array}{cc}
\pi / K & 0 \\
0 & 2 / P
\end{array}\right)
$$

where $K$ and $P$ satisfy the sampling conditions $b \leq K \vartheta, b \leq$ $\pi P / 2$ (see Fig. 2 left). The optimal choice, i.e., the minimal value for $K$ and $P$ is $\pi P / 2=b ; 2 K=\pi P / \vartheta$.

$$
W_{I}=\left(\begin{array}{cc}
2 \pi / K & -\pi / K \\
0 & 2 / P
\end{array}\right)
$$

where $2 K=\pi P / \vartheta^{\prime}, b \leq \pi P / 2$ (see Fig. 2 right). We remark that $\operatorname{det} W_{I} \approx 2 \operatorname{det} W_{S}$ (it is in fact a bit less than $2 \operatorname{det} W_{S}$ because $\vartheta^{\prime}<\vartheta$-see Fig. 2). Thus, the interlaced lattice elementary mesh area is almost twice as large as that of the standard one. This means that the same goal can be obtained with two times less data. The sets $\mathbf{S}+2 \pi\left(W_{I}\right)^{-t} k, k \in \mathbb{Z}^{2}$ almost tile the Fourier plane: This shows that the interlaced scheme is an optimal scheme for $\mathbf{S}$.

The previous sampling schemes are also valid in vector tomography with a small adaptation. The results of 
Section II-B yield a widening of $\mathbf{S}$ becoming $\mathbf{S}^{\prime}=$ $\{(m, \sigma) ;|\sigma|<b,|m|<1+\max (|\sigma| / \vartheta,(1 / \vartheta-1) b\}, \quad$ and thus, the vectors $\vec{u}_{1}$ and $\overrightarrow{u_{1}^{\prime}} 1$ in Fig. 2 only have to be replaced by $\overrightarrow{u^{\prime \prime}}{ }_{1}$ and $\overrightarrow{u^{\prime \prime \prime}}{ }_{1}$ with respective components $(2(1+b / \vartheta), 0)^{t}$ and $\left(2+b / \vartheta^{\prime}, b\right)^{t}$. Choosing $\vartheta^{\prime \prime}$ and $\vartheta^{\prime \prime \prime}$ such that $b / \vartheta^{\prime \prime}=1+b / \vartheta, b / \vartheta^{\prime \prime \prime}=2+b / \vartheta^{\prime}$, i.e., $\vartheta^{\prime \prime}=\frac{b}{b+\vartheta} \vartheta$ and $\vartheta^{\prime \prime \prime}=\frac{b}{b+2 \vartheta^{\prime}} \vartheta^{\prime}$, we obtain the same sampling conditions replacing $\vartheta$ by $\vartheta^{\prime \prime}$ in the standard case and $\vartheta^{\prime}$ by $\vartheta^{\prime \prime \prime}$ in the interlaced case. The number of projections only has to be slightly greater (one more) in each case.

We remark that $K$ must be even in the interlaced scheme for using the symmetry of the Radon transform: $\mathcal{R} f(\theta, s)=$ $\mathcal{R} f(\theta+\pi,-s)$. If not, the same data are used as in the standard scheme.

For generalizations on efficient sampling schemes, see [5], [8], and [9]. For efficient fan-beam sampling schemes, see [14].

\section{Direct Algebraic APPROACH}

\section{A. Introduction}

Let us take the following model for the data in vector field tomography: For $k=0, \cdots, K-1, p=1, \cdots, P$,

$$
\begin{aligned}
\mathbf{y}_{k, p} & =\int_{L_{\theta_{k}, s_{p}} \cap \Omega} v_{2} \cos \phi_{k}-v_{1} \sin \phi_{k} d \Omega \\
& =\int_{\Omega}\left\langle\tau_{k} \mathbb{1}_{L_{\theta_{k}, s_{p}},} \vec{v}\right\rangle_{\mathbb{R}^{2}},
\end{aligned}
$$

where $v$ is the field vector in the cross section called the $\Omega$ domain, which will be the unit disk. $\theta_{k}=\left(\cos \phi_{k}, \sin \phi_{k}\right)$ and $\tau_{k}=\left(-\sin \phi_{k}, \cos \phi_{k}\right)$ are orthogonal unitary vectors, and $L_{\theta_{k}, s_{p}}$ is a strip centered on $s_{p}$ of constant width $e$ orthogonal to the direction $\theta_{k}$ (see Fig. 3). We will denote $L_{k, p} \stackrel{\text { def }}{=} L_{\theta_{k}, s_{p}}$ and

$$
\begin{aligned}
l_{k, p}(x) & =\mathbb{1}_{L_{k, p}}(x) \\
& = \begin{cases}1 & \text { if } x \in L_{k, p}, \text { i.e., }\left|\left\langle x, \theta_{k}\right\rangle_{\mathbb{R}^{2}}-s_{p}\right|<e / 2 \\
0 & \text { otherwise }\end{cases}
\end{aligned}
$$

In addition, we will call $K \times P$ geometries covering the unit disk $\Omega$ "standard" when they verify $\phi_{k}=k \pi / K, k=$ $0, \cdots, K-1, s_{p}=(2 p-1-P) / P, p=1, \cdots, P, e=2 / P$.

Let us denote $\mathcal{R}$ the continuous linear operator between the two following Hilbert spaces:

$$
\begin{aligned}
\mathcal{R}: & \left(L^{2}(\Omega)\right)^{2} \\
\vec{f}\left(=\left(f_{1}, f_{2}\right)^{t}\right) & \longrightarrow \mathbb{R}^{K P} \\
& \longrightarrow \int_{L_{k, p}}\left\langle\tau_{k}, \vec{f}\right\rangle_{\mathbb{R}^{2}} .
\end{aligned}
$$

The associated least square problem

$$
\min _{\vec{f} \in\left(L^{2}(\Omega)\right)^{2}}\|\mathcal{R} \vec{f}-\mathbf{y}\|^{2}
$$

has the following minimal norm solution:

$$
\overrightarrow{f^{\dagger}}=\mathcal{R}^{*} \mathbf{f}^{\dagger}, \text { where } \mathbf{f}^{\dagger}=\left(\mathcal{R} \mathcal{R}^{*}\right)^{\dagger} \mathbf{y}
$$

where $\left(\mathcal{R} \mathcal{R}^{*}\right)^{\dagger}$ designates the generalized inverse (or Moore-Penrose inverse; see [1]). In this case

$$
\begin{aligned}
\mathcal{R}^{*}: \mathbb{R}^{K P} & \longrightarrow\left(L^{2}(\Omega)\right)^{2} \\
\mathbf{y} & \longrightarrow \sum_{k, p} \mathbf{y}_{k, p} l_{k, p}(x) \tau_{k} .
\end{aligned}
$$

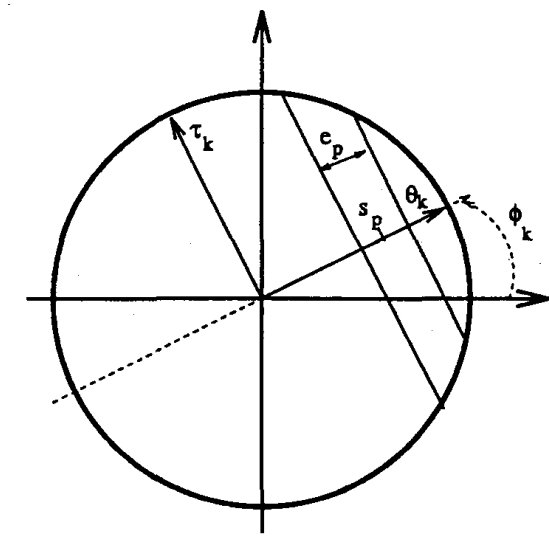

Fig. 3. Measurement geometry. In the DA approach, $\langle\vec{v}, \tau\rangle$ is integrated over strips.

Indeed

$$
\begin{aligned}
\left\langle\mathcal{R}^{*} \mathbf{y}, \vec{f}\right\rangle_{\left(L^{2}\right)^{2}} & =\langle\mathbf{y}, \mathcal{R} \vec{f}\rangle_{\mathbb{R}^{K P}} \\
& =\sum_{k, p} \mathbf{y}_{k, p} \int_{L_{k, p}}\left\langle\tau_{k}, \vec{f}\right\rangle_{\mathbb{R}^{2}} \\
& =\int_{\Omega}\left\langle\sum_{k, p} \mathbf{y}_{k, p} l_{k, p} \tau_{k}, \vec{f}\right\rangle_{\mathbb{R}^{2}} .
\end{aligned}
$$

Thus, $\mathcal{R R}^{*}$ has the following entries:

$$
\begin{aligned}
\left(\mathcal{R} \mathcal{R}^{*}\right)_{k^{\prime}, p^{\prime}, k, p} & =\left\langle\tau_{k}, \tau_{k^{\prime}}\right\rangle_{\mathbb{R}^{2}} \int_{\Omega} l_{k, p}(x) l_{k^{\prime}, p^{\prime}}(x) d x \\
& =\cos \left(\phi_{k}-\phi_{k^{\prime}}\right) \int_{\Omega} l_{k, p}(x) l_{k^{\prime}, p^{\prime}}(x) d x .
\end{aligned}
$$

Thus, the matrix $\mathcal{R R}^{*}$ has the same entries as the scalar tomography matrix in the same DA approach $\left(f \in L^{2}\right)$, except for the new term $\cos \left(\phi_{k}-\phi_{k^{\prime}}\right)$. Thus, with a small change, a scalar tomography code can be used for computing $\mathrm{f}^{\dagger}$. The vector backprojection operator $\mathcal{R}^{*}$ is essentially the same as the scalar operator except for the new vector term $\tau_{k}$.

\section{B. $\mathcal{R} \mathcal{R}^{*}$ Kernel for Standard Geometries}

Let us denote the canonical basis $e_{i, j} \in \mathbb{R}^{K P}$, i.e., $\left(e_{i, j}\right)_{k, p}=\delta_{i k} \delta_{j p}, i=0 \cdots, K-1 ; j=1, \cdots, P$. We denote $e_{i} \stackrel{\text { def }}{=} \sum_{j=1}^{P} e_{i, j}$. We have

$$
\mathcal{R}^{*} e_{k}=\sum_{p=1}^{P} l_{k, p} \tau_{k}=\mathbb{1}_{\Omega} \tau_{k}, \forall k=0, \cdots, K-1 .
$$

We thus have the following proposition.

Proposition 1: For standard geometries and $K>2$,

$$
\begin{aligned}
\operatorname{ker} \mathcal{R}^{*} & \left.=\operatorname{ker} \mathcal{R} \mathcal{R}^{*}\right) . \\
& \left.=\mathcal{L}\left(\left(2 \cos \phi_{1} e_{k}-e_{k-1}-e_{k+1}\right)\right)_{k=1, \cdots, K-2}\right) .
\end{aligned}
$$

Notice the difference with the usual tomography optimal algorithm matrices: $\operatorname{dim}\left(\operatorname{ker} \mathcal{R}^{*}\right)=K-2$ (instead of $K-$ 1 ; see [12]). One of the practical uses of this proposition is the possibility of testing the correctness of the matrix entries computation algorithm. The first part of the proof is Proposition 2. 


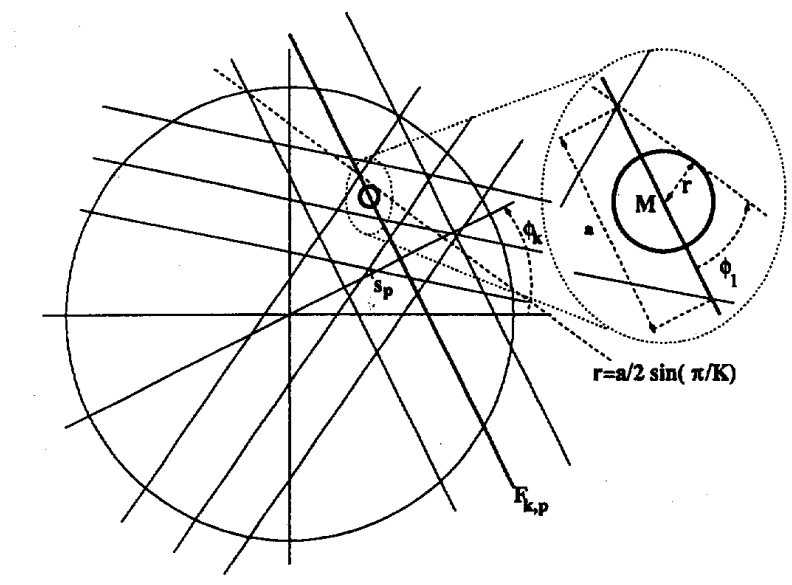

Fig. 4. In each direction $k$, for each frontier $F_{k, p}$ between two strips $p$ and $p+1$, there are two nonempty regions (e.g., two half disks) separated by $F_{k, p}$ such that both regions are covered by the same strips in all other directions.

Proposition 2: For standard geometries $\operatorname{ker} \mathcal{R}^{*} \quad \subset$ $\mathcal{L}\left(e_{i}\right)_{i=0, \cdots, K-1}$.

Proof: The proof is adapted from [12]. $\forall k=0, \cdots, K-$ $1, \forall p=1, \ldots, P-1$, consider the frontier $F_{k, p}$ between two strips $p$ and $p+1$ in the direction $\phi_{k}$ (see Fig. 4). The strip frontiers in the other directions produce intersection points with $F_{k, p}$. Take two different successive intersection points in $\Omega$ distant from $a(\neq 0)$, let $M$ be their middle point. Then, $\forall x, x^{\prime} \in \mathcal{B}_{k, p}(M, a / 2 \sin \pi / K)(\subset \Omega)$, i.e., the disk centered on $M$ of radius $a / 2 \sin \pi / K, \forall \mathbf{y} \in \mathbb{R}^{K P}$

$$
\begin{aligned}
\mathcal{R}^{*} \mathbf{y}(x)- & \mathcal{R}^{*} \mathbf{y}\left(x^{\prime}\right)=\left(\mathbf{y}_{k, p+1}-\mathbf{y}_{k, p}\right) \\
& \cdot\left(l_{k, p+1}(x) l_{k, p}\left(x^{\prime}\right)-l_{k, p}(x) l_{k, p+1}\left(x^{\prime}\right)\right) \tau_{k} .
\end{aligned}
$$

$\mathcal{R}^{*} \mathbf{y}(x)$ is constant on each half of the disk $\mathcal{B}_{k, p}$ separated with the line $F_{k, p}$. The value of $\mathcal{R}^{*} y$ for two points, each of them in a different half of the disk, differs only through $\mathbf{y}_{k, p+1}$ and $\mathbf{y}_{k, p}$. Thus $\mathbf{y} \in \operatorname{ker} \mathcal{R}^{*} \Rightarrow \forall k, \forall p, \forall x \in \mathcal{B}_{k, p}(M, a / 2 \sin \pi / K) \cap L_{k, p+1}$, $\forall x^{\prime} \in \mathcal{B}_{k, p}(M, a / 2 \sin \pi / K) \cap L_{k, p+1}, \mathcal{R}^{*} \mathbf{y}(x)=\mathcal{R}^{*} \mathbf{y}\left(x^{\prime}\right)(=$ $0)$, and thus $\mathbf{y}_{k, p+1}-\mathbf{y}_{k, p}=0$.

Proof of Proposition 1: We have $\cos \phi_{1} \cos \phi_{k}=$ $1 / 2\left(\cos \phi_{k+1}+\cos \phi_{k-1}\right)$ and $\cos \phi_{1} \sin \phi_{k}=1 / 2\left(\sin \phi_{k+1}+\right.$ $\sin \phi_{k-1}$ ) or, $2 \cos \phi_{1} \tau_{k}=\tau_{k+1}+\tau_{k-1}$. Thus, from (9), $\forall k=1, \cdots, K-2, \mathcal{R}^{*}\left(2 \cos \phi_{1} e_{k}-e_{k-1}-e_{k+1}\right)=0$. We also have $\mathcal{R}^{*}\left(2 \cos \phi_{1} e_{0}+e_{K}-e_{1}\right)=0$ and $\mathcal{R}^{*}\left(2 \cos \phi_{1} e_{K}-e_{K-1}+e_{0}\right)=0$.

Let us consider the following $K \times K$ matrix representing a linear operator in $\mathcal{L}\left(e_{k}\right)_{k=0, \cdots, K-1}$ :

$$
M=\left[\begin{array}{cccccc}
2 \cos \phi_{1} & -1 & 0 & \ldots & 0 & 1 \\
-1 & 2 \cos \phi_{1} & -1 & 0 & \cdots & 0 \\
0 & \ddots & \ddots & \ddots & \ddots & \vdots \\
\vdots & \ddots & \ddots & \ddots & \ddots & 0 \\
0 & \cdots & 0 & -1 & 2 \cos \phi_{1} & -1 \\
1 & 0 & \cdots & 0 & -1 & 2 \cos \phi_{1}
\end{array}\right] .
$$

The rank of $M$ is $K-2$. The $K-2$ vectors $2 \cos \phi_{1} e_{k}-e_{k-1}-$ $e_{k+1}, k=1, \cdots, K-2$ are obviously linearly independent. Its kernel is reduced to the plane generated by the two orthogonal vectors $\left(\cos \phi_{k}\right)_{k=0, \cdots, K-1},\left(\sin \phi_{k}\right)_{k=0, \cdots, K-1}$. We have

$$
\begin{aligned}
& \mathcal{R}^{*}\left(\lambda \sum_{k=0}^{K-1} \cos \phi_{k} e_{k}+\mu \sum_{k=0}^{K-1} \sin \phi_{k} e_{k}\right)=0 \\
& \Rightarrow \lambda \sum_{k=0}^{K-1} \cos \phi_{k} \tau_{k}+\mu \sum_{k=0}^{K-1} \sin \phi_{k} \tau_{k}=0 \\
& \Rightarrow\left(-\mu \sum_{k=0}^{K-1} \sin ^{2} \phi_{k} ; \lambda \sum_{k=0}^{K-1} \cos ^{2} \phi_{k}\right)=0 \\
& \Rightarrow \lambda=\mu=0 .
\end{aligned}
$$

As $\mathcal{L}\left(e_{k}\right)_{k=0, \cdots, K-1}=\operatorname{ker} M \oplus \operatorname{range} M$, we can conclude from Proposition 2 using $\mathcal{R}^{*} M=0$ in $\mathcal{L}\left(e_{k}\right)_{k=0, \cdots, K-1}$ and $\mathbf{x} \in \operatorname{ker} M, \mathcal{R}^{*} \mathbf{x}=0 \Rightarrow \mathbf{x}=0$ that

$$
\begin{aligned}
\operatorname{ker} \mathcal{R}^{*} & =\text { range } M \\
& =\mathcal{L}\left(\left(2 \cos \phi_{1} e_{k}-e_{k-1}-e_{k+1}\right)_{k=1, \cdots, K-2}\right) .
\end{aligned}
$$

\section{EFFICIENT ALGORITHMS}

\section{A. Standard Geometries}

Efficient DA algorithms are based on rotational invariance of the measurement geometry. From (8), we have

$$
\mathcal{R} \mathcal{R}_{k, p, k^{\prime}, p^{\prime}}^{*}=\mathcal{R} \mathcal{R}_{k-k^{\prime}, p, 0, p^{\prime}}^{*}
$$

Thus, we can reorganize measurement in order to obtain a block circulant matrix for $\mathcal{R \mathcal { R }}^{*}: \forall p=1, \cdots,\lceil P / 2\rceil$,

$$
l_{k, p}^{e} \tau_{k}= \begin{cases}l_{k,\lfloor P / 2\rfloor+p} \tau_{k} & k=0, \cdots, K-1 \\ -l_{k-K,\lceil P / 2\rceil-p+1} \tau_{k-K} & k=K, \cdots, 2 K-1\end{cases}
$$

where $\lfloor r\rfloor$ is the greatest integer smaller than or equal to $r$, and $\lceil r\rceil$ is the smallest integer greater or equal to $r$. Note that the main difference with the well-known natural pixels in scalar tomography is the antisymmetry $\tau_{k+K}=-\tau_{k}$. Note that if $P$ is odd, the center strip is used twice to get the rotational invariance. Thus, for solving the original least squares problem, we have to weigh the corresponding measurement with a factor $1 / \sqrt{2}$. In this case, the number of null eigenvalues of the matrix $\mathcal{R}^{e} \mathcal{R}^{e *}$ is $2 K-2$.

$$
\mathcal{R}^{e} \mathcal{R}^{e *}=\left[\begin{array}{cccc}
R^{0} & R^{2 K-1} & \ldots & R^{1} \\
R^{1} & R^{0} & \ddots & \vdots \\
\vdots & \ddots & \ddots & R^{2 K-2} \\
R^{2 K-1} & \ldots & R^{1} & R^{0}
\end{array}\right]
$$

where $R_{p, q}^{k}=\cos \phi_{k}\left\langle l_{k, p}^{e}, l_{0, q}^{e}\right\rangle_{L^{2}(\Omega)}, p=1, \cdots, \frac{P}{2}, q=$ $1, \cdots, \frac{P}{2}$. As for the usual direct algorithm in scalar tomography, we have $R^{k}=R^{2 K-k}, \forall k=1, \cdots, K$ because $\cos ((2 K-k) \pi / K)=\cos (k \pi / K)$ and the obvious geometrical symmetry according to the $x_{1}$ axis of the functions $l_{k, p}^{e}$ and $l_{2 K-k, p}^{e}$. Consequently, $R^{k}=\left(R^{k}\right)^{t}$, and thus, $R_{p, q}^{k}=\cos \phi_{k}\left\langle l_{0, p}^{e}, l_{2 K-k, q}^{e}\right\rangle_{L^{2}(\Omega)} . R^{K}=0$ if $P$ is even. Otherwise, since the central strip is repeated when $P$ is odd, $R_{1,1}^{K} \neq 0$. In vector tomography, if $K$ is even, we also 
have $R^{K / 2}=R^{3 K / 2}=0(\cos (\pi / 2)=\cos (3 \pi / 2)=0)$. The minimal norm solution (7) can be efficiently computed using Fourier transforms (see [12], [3], [10]). For $v \in \mathbb{R}^{2 K\lceil P / 2\rceil}$, we denote $\mathbf{v}^{k} \in \mathbb{R}^{\lceil P / 2\rceil}$ for the $2 K$ vectors such that $v_{p}^{k}=v_{k, p}$ and let $M^{j}$ be $2 K$ matrix $\lceil P / 2\rceil \times\lceil P / 2\rceil$; then, we consider the following Fourier transformation:

$$
\hat{\mathbf{v}}_{p}^{k} \stackrel{\text { def }}{=} \sum_{j=0}^{2 K-1} e^{-2 \pi i j k / 2 K} v_{p}^{j} \text { and } \hat{M}_{p, q}^{k} \stackrel{\text { def }}{=} \sum_{j=0}^{2 K-1} e^{-2 \pi i j k / 2 K} M_{p, q}^{j} .
$$

We have

$$
\mathcal{R}^{e} \mathcal{R}^{e *} \mathbf{f}^{e}=\mathbf{y}^{e} \Leftrightarrow \hat{R}^{k}\left(\hat{\mathbf{f}^{e}}\right)^{k}=\left(\hat{\mathbf{y}}^{e}\right)^{k}, \forall k=0, \cdots, 2 K-1 .
$$

These $2 K$ linear systems of order $[P / 2\rceil$ are generally solved using singular value decompositions producing an algorithm in $O\left(K P^{3}\right)$. Spectrum truncation (see [11] for an efficient and elegant choice of the parameter) or regularization methods are generally used (see [10]) to copmpute a stable solution of these normal equations. If the geometry is fixed and the stabilized pseudo-inverse $\left(\hat{R}^{k}\right)^{\dagger}$ stored, the computation of a solution is in $O\left(K P^{2}\right)$.

\section{B. Interlaced Geometries}

1) Introduction: In the algebraic approach, the interlaced scheme corresponds to the following choice of half of the standard measurement functions. For $k=0, \cdots, 2 K-1$

$$
l_{k, p}^{+, o}=\left\{\begin{array}{ll}
l_{k, 2 p-1}^{e} & \text { if } k \text { even, for } p=1, \cdots,\left\lceil\frac{P}{4}\right\rceil \\
l_{k, 2 p}^{e} & \text { if } k \text { odd, for } p=1, \cdots,\left\lceil\frac{P}{2}\right\rceil-\left\lceil\frac{P}{4}\right\rceil
\end{array} .\right.
$$

The second possible interlaced scheme is simply the complementary scheme of 10 for the corresponding standard scheme:

$$
l_{k, p}^{-, o}= \begin{cases}l_{k, 2 p-1}^{e} & \text { if } k \text { odd, for } p=1, \cdots,\left\lceil\frac{P}{4}\right\rceil \\ l_{k, 2 p}^{e} & \text { if } k \text { even, for } p=1, \cdots,\left\lceil\frac{P}{2}\right\rceil-\left\lceil\frac{P}{4}\right\rceil\end{cases}
$$

Remark that when $P$ is odd, the points $\left(\phi_{k}, s_{p}\right)^{t}$ of the interlaced geometry can be written $W_{I}\left(k^{\prime}, p^{\prime}\right)^{t}$ for some integers $k^{\prime}$ and $p^{\prime}$. Thus, as can be seen in Section II-C, $K$ has to be even to result in an efficient interlaced scheme, i.e., in order to make use of the Radon transform symmetry and to avoid dealing with the standard data when $\phi$ goes from 0 to $2 \pi$. When $P$ is even, the points $\left(\phi_{k}, s_{p}\right)^{t}$ of the interlaced geometry can be written $(0,1 / P)^{t}+W_{I}\left(k^{\prime}, p^{\prime}\right)^{t}$ for some integers $k^{\prime}$ and $p^{\prime}$. $K$ must then be odd to obtain an interlaced sampling, i.e., to use the Radon transform symmetry. Thus, $K$ and $P$ must have a different parity in the case of interlaced schemes. This plays an important role in the decomposition of the DA matrix presented in the next section in Proposition 4.

2) An Efficient Robust Algorithm: The first ideas for processing interlaced data with the direct algebraic approach came from Klaverkamp [11]. In the following, we adapted a different algorithm proposed in [7] to vector tomography. A complete proof of the least-squares matrix decomposition into a real block-rectangular matrix is given.

Interlaced data give the same reconstruction as that obtained with twice as much data in the corresponding standard scheme. Thus, a natural way to formulate the reconstruction problem is to seek the best approximation of $f$ in $\operatorname{range}\left(\mathcal{R}^{*}\right)$ from the interlaced data. As in Section IV-A, we first reorganize the measurement in order to get an invariant rotational scheme. From (10), we denote for $k=0, \cdots, K-1$ :

$$
l_{k, p}^{+}=\left\{\begin{array}{ll}
l_{2 k, p}^{+, o} & \text { for } p=1, \cdots,\left\lceil\frac{P}{4}\right\rceil \\
l_{2 k+1, p-\left\lceil\frac{P}{4}\right\rceil}^{+, o} & \text { for } p=\left\lceil\frac{P}{4}\right\rceil+1, \cdots,\left\lceil\frac{P}{2}\right\rceil .
\end{array} .\right.
$$

Note that now, $\tau^{+}{ }_{k(p)}$ depends on $p$ :

$$
\tau_{k(p)}^{+}=\left\{\begin{array}{ll}
\tau_{2 k} & \text { for } p=1 \cdots,\left\lceil\frac{P}{4}\right\rceil \\
\tau_{2 k+1} & \text { for } p=\left\lceil\frac{P}{4}\right\rceil+1, \cdots,\left\lceil\frac{P}{2}\right\rceil
\end{array} .\right.
$$

We also derive from (11)

$$
\begin{aligned}
& l_{k, p}^{-}= \\
& \begin{cases}l_{2 k, p}^{-, o} & \text { for } p=1, \cdots,\left\lceil\frac{P}{2}\right\rceil-\left\lceil\frac{P}{4}\right\rceil \\
l_{2 k+1, p-\left\lceil\frac{P}{2}\right\rceil+\left\lceil\frac{P}{4}\right\rceil}^{-, o} & \text { for } p=\left\lceil\frac{P}{2}\right\rceil-\left\lceil\frac{P}{4}\right\rceil+1, \cdots,\left\lceil\frac{P}{2}\right\rceil\end{cases}
\end{aligned}
$$

$$
\tau^{-}{ }_{k(p)}=\left\{\begin{array}{ll}
\tau_{2 k} & \text { for } p=1, \cdots,\left\lceil\frac{P}{2}\right\rceil-\left\lceil\frac{P}{4}\right\rceil, \\
\tau_{2 k+1} & \text { for } p=\left\lceil\frac{P}{2}\right\rceil-\left\lceil\frac{P}{4}\right\rceil+1, \cdots,\left\lceil\frac{P}{2}\right\rceil .
\end{array} .\right.
$$

Since we seek the solution $f \in \operatorname{range}\left(\mathcal{R}^{*}\right)$, we write

$$
f^{\dagger}=\sum_{k^{\prime}=0}^{2 K-1} \sum_{p^{\prime}=1}^{\left\lceil\frac{P}{2}\right\rceil} \mathbf{f}_{k^{\prime}, p^{\prime}} l_{k^{\prime}, p^{\prime}}^{e} \tau_{k}
$$

Thus, we want to solve

$$
\begin{aligned}
& \min _{\mathbf{f} \in \mathbb{R}^{K P}} \sum_{k=0}^{K-1} \sum_{p=1}^{\left\lceil\frac{P}{2}\right\rceil} \\
& \left(\left\langle l_{k, p}^{+} \tau^{+} k(p), \sum_{k^{\prime}=0}^{K-1} \sum_{p^{\prime}=1}^{\left\lceil\frac{P}{2}\right\rceil} \mathbf{f}_{k^{\prime}, p^{\prime}} l_{k^{\prime}, p^{\prime}}^{e} \tau_{k^{\prime}}\right\rangle_{\left(L^{2}(\Omega)\right)^{2}}-\mathbf{y}_{k, p}^{+}\right)^{2} .
\end{aligned}
$$

We can reorganize the strip functions $l_{k, p}^{e}$ of $\mathcal{R}^{*}$ according to both the interlaced schemes underlying the standard one. From (12) and (13), we have

$$
\begin{aligned}
& \sum_{k^{\prime}=0}^{2 K-1} \sum_{p^{\prime}=1}^{\left\lceil\frac{P}{2}\right\rceil} \mathbf{f}_{k^{\prime}, p^{\prime}} l_{k^{\prime}, p^{\prime}}^{e} \tau_{k}= \\
& \sum_{k^{\prime}=0}^{K-1} \sum_{p^{\prime}=1}^{\left\lceil\frac{P}{2}\right\rceil} \mathbf{f}_{k^{\prime}, p^{\prime}}^{+} l_{k^{\prime}, p^{\prime}}^{+} \tau^{+}{ }_{k^{\prime}\left(p^{\prime}\right)}+\mathbf{f}_{k^{\prime}, p^{\prime}}^{-}, l_{k^{\prime}, p^{\prime}}^{-} \tau^{-}{ }_{k^{\prime}\left(p^{\prime}\right)}
\end{aligned}
$$

Equation (14) is equivalent to the first equation at the bottom of the next page.

The $K\lceil P / 2\rceil \times 2 K\lceil P / 2\rceil$ matrix of the last least squares problem is denoted $\left(R^{+}, R^{-}\right)$and has the regular structure that is shown as the second equation at the bottom of the next page, where $R^{\epsilon, k}$ is a $\left\lceil\frac{P}{2}\right\rceil \times\left\lceil\frac{P}{2}\right\rceil$ matrix such that $R_{p, p^{\prime}}^{\epsilon, k}=\left\langle\tau_{k(p)}^{+}, \tau_{0\left(p^{\prime}\right)}^{\epsilon}\right\rangle_{\mathbb{R}^{2}}\left\langle l_{k, p}^{+}, l_{0, p^{\prime}}^{\epsilon}\right\rangle_{L^{2}(\Omega)}(\epsilon \in\{+,-\})$. Note that $\left\langle\tau_{k(p)}^{+}, \tau_{0\left(p^{\prime}\right)}^{\epsilon}\right\rangle_{\mathbb{R}^{2}}$ takes the value $\cos \phi_{2 k}$ for the blocks $R^{\epsilon, k, 00}$ and $R^{\epsilon, k, 11}, \cos \phi_{2 k-1}$ for the block $R^{\epsilon, k, 01}$, and $\cos \phi_{2 k+1}$ for the block $R^{\epsilon, k, 10}$, cf. (15). Both circulant parts can be block diagonalized by discrete Fourier transforms of 
order $K$ yielding the following $K$ independent least squares problems:

$\min _{\hat{\mathbf{f}}^{+, k}, \hat{\mathbf{f}}^{-, k}}\left\|\hat{R}^{+, k} \hat{\mathbf{f}}^{+, k}+\hat{R}^{-, k} \hat{\mathbf{f}}^{-, k}-\hat{\mathbf{y}}^{+, k}\right\|^{2}, \forall k=0, \cdots, K-1$

where $\mathbf{y}_{p}^{+, k}$ is simply $\mathbf{y}_{k, p}^{+}$and $\mathbf{f}_{p}^{\epsilon, k}=\mathbf{f}_{k, p}^{\epsilon}$.

In the following, we show that the complex matrix $\hat{R}^{\epsilon, k}$ can be simply transformed into a real matrix. The matrices $R^{\epsilon, k}$ and $\hat{R}^{\epsilon, k}$ are written as

$$
R^{\epsilon, k}=\left(\begin{array}{ll}
R^{\epsilon, k, 00} & R^{\epsilon, k, 01} \\
R^{\epsilon, k, 10} & R^{\epsilon, k, 11}
\end{array}\right), \quad \hat{R}^{\epsilon, k}=\left(\begin{array}{ll}
\hat{R}^{\epsilon, k, 00} & \hat{R}^{\epsilon, k, 01} \\
\hat{R}^{\epsilon, k, 10} & \hat{R}^{\epsilon, k, 11}
\end{array}\right) .
$$

$\hat{R}^{+, k, 00}$ and $\hat{R}^{+, k, 11}$ are real symmetric square matrices of dimension $\left\lceil\frac{P}{4}\right\rceil$ and $\left\lceil\frac{P}{2}\right\rceil-\left\lceil\frac{P}{4}\right\rceil$, respectively, produced by circulant schemes for which $\phi_{1}=2 \pi / K . \hat{R}^{-, k, 00}$ and $\hat{R}^{-, k, 11}$ are real matrices of dimension $\left[\frac{P}{4}\right\rceil \times\left(\left\lceil\frac{P}{2}\right\rceil-\left[\frac{P}{4}\right\rceil\right)$ and $\left(\left\lceil\frac{P}{2}\right\rceil-\left\lceil\frac{P}{4}\right\rceil\right) \times\left\lceil\frac{P}{4}\right\rceil$, respectively, for the same reason. Note that the $\hat{R}^{-, k, x x}$ matrices are not symmetric and are not even square when $\lceil P / 2\rceil$ is odd.

Proposition 3:

$$
\hat{R}^{\epsilon, k, 10}=e^{\frac{i k \pi}{K}} S^{\epsilon, k, 10} \text { and } \hat{R}^{\epsilon, k, 01}=e^{\frac{-i k \pi}{K}} S^{\epsilon, k, 01}
$$

where $S^{\epsilon, k, 10}$ and $S^{\epsilon, k, 01}$ are real matrices, $S^{\epsilon, K-k, 10}=$ $-S^{\epsilon, k, 10}, S^{\epsilon, K-k, 01}=-S^{\epsilon, k, 01}$. Moreover, $S^{+, k, 10}=$ $\left(S^{+, k, 01}\right)^{t}$. Below $S^{+, k, 10}$ is denoted $S^{k}$.

We will make use of the obvious lemma.

Lemma 1: Let $a_{j} \in \mathbb{R}, j=0, \cdots, K-1$ such that $a_{j}=a_{K-1-j}$; then

$$
\hat{a}_{k}=e^{\frac{i k \pi}{K}} s_{k} \text { where } s_{k} \in \mathbb{R} \text { and } s_{K-k}=-s_{k} .
$$

From Lemma 1 , we also have $a_{j}, j=0, \cdots, K-1$ such that $a_{0}=a_{1}, a_{2+j}=a_{K-1-j}$; then, $\hat{a}_{k}=e^{\frac{-i k \pi}{K}} s_{k}^{\prime}$ where $s_{k}^{\prime} \in$ $\mathbb{R}$ and $s_{K-k}^{\prime}=-s_{k}$.

Proof of Proposition 3: In order to apply Lemma 1, we now simply verify (17) and (18):

- First:

$$
R_{p, q}^{\epsilon, j, 10}=R_{p, q}^{\epsilon, K-1-j, 10}
$$

Indeed, let us introduce $\mu(\epsilon)=1$ if $\epsilon=+$, or 0 otherwise. We have

$$
R_{p, q}^{\epsilon, j, 10}=\cos \frac{(2 j+1) \pi}{K}\left\langle l_{2 j+1,2 p}^{e}, l_{0,2 q-\mu(\epsilon)}^{e} Z\right\rangle_{L^{2}(\Omega)}
$$

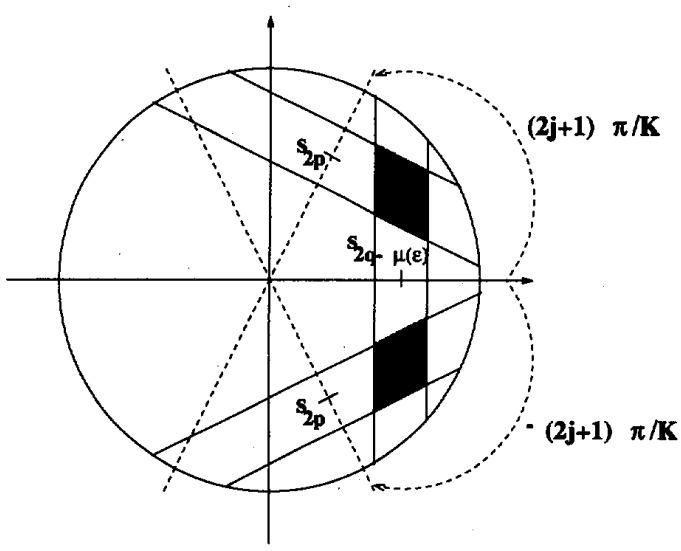

Fig. 5. $\quad\left\langle l_{2 j+1,2 p}^{e} l_{0,2 q-\mu(\epsilon)}^{e}\right\rangle_{L^{2}(\Omega)}=\left\langle l_{2 K-2 j-1,2 p}^{e} l_{0,2 q-\mu(\epsilon)}^{e}\right\rangle_{L^{2}(\Omega)}$ :

and thus

$$
\begin{aligned}
R_{p, q}^{\epsilon, K-1-j, 10}= & \cos \frac{(2 K-2 j-1) \pi}{K} \\
& \left\langle l_{2 K-2 j-1,2 p}^{e}, l_{0,2 q-\mu(\epsilon)}^{e}\right\rangle_{L^{2}(\Omega)} .
\end{aligned}
$$

As $\cos \frac{(2 K-2 j-1) \pi}{K}=\cos \frac{(2 j+1) \pi}{K}$ and $\left(l_{2 j+1,2 p}^{e}\right.$ $l_{0,2 q-\mu(\epsilon)}^{e} L^{2}(\Omega)=\left\langle l_{2 K-2 j-1,2 p}^{e}, l_{0,2 q-\mu(\epsilon)}^{e}\right\rangle_{L^{2}(\Omega)}$ for obvious geometrical reasons (see Fig. 5), (17) is shown. $S^{+, k, 10}=\left(S^{+, k, 01}\right)^{t}$ because $\hat{R}^{+, k}$ is Hermitian. Indeed, $R^{+}$is the Gramm matrix corresponding to the $l_{k p}^{+}$ functions and is thus symmetric.

- Second:

$$
R_{p, q}^{\epsilon, 0,01}=R_{p, q}^{\epsilon, 1,01}, R_{p, q}^{\epsilon, j+2,01}=R_{p, q}^{\epsilon, K-1-j, 01} .
$$

Indeed

$$
R_{p, q}^{\epsilon, j, 01}=\cos \frac{(2 j-1) \pi}{K}\left\langle l_{2 j, 2 p-1}^{e}, l_{1,2 q-\mu(\epsilon)}^{e}\right\rangle_{L^{2}(\Omega)} .
$$

We have $\cos \frac{(2(K-1-j)-1) \pi}{K}=\cos \frac{(2(j+2)-1) \pi}{K}$ as well as

$$
\begin{aligned}
& \left\langle l_{2(j+2), 2 p-1}^{e}, l_{1,2 q-\mu(\epsilon)}^{e}\right\rangle_{L^{2}(\Omega)} \\
& =\left\langle l_{2(K-1-j), 2 p-1}^{e}, l_{1,2 q-\mu(\epsilon)}^{e}\right\rangle_{L^{2}(\Omega)}
\end{aligned}
$$

for obvious geometrical reasons (see Fig. 6), and thus, (18) is shown.

$$
\min _{\mathbf{f}^{+}, \mathbf{f}^{-}} \sum_{k=0}^{K-1} \sum_{p=1}^{\left\lceil\frac{P}{2}\right\rceil}\left(\left\langle l_{k, p}^{+} \tau^{+} k(p), \sum_{k^{\prime}=0}^{K-1} \sum_{p^{\prime}=1}^{\left\lceil\frac{P}{2}\right\rceil} \mathbf{f}_{k^{\prime}, p^{\prime}}^{+} l_{k^{\prime}, p^{\prime}}^{+} \tau^{+}{ }_{k^{\prime}\left(p^{\prime}\right)}+\sum_{k^{\prime}=0}^{K-1} \sum_{p^{\prime}=1}^{\left\lceil\frac{P}{2}\right\rceil} \mathbf{f}_{k^{\prime}, p^{\prime}}^{-} l_{k^{\prime}, p^{\prime}}^{-} \tau^{-}{ }_{k^{\prime}\left(p^{\prime}\right)}\right\rangle_{L_{2}^{2}(\Omega)}-\mathbf{y}_{k, p}^{+}\right)^{2}
$$

$$
\left(\begin{array}{cccccccc}
R^{+, 0} & R^{+, K-1} & \ldots & R^{+, 1} & R^{-, 0} & R^{-, K-1} & \ldots & R^{-, 1} \\
R^{+, 1} & R^{+, 0} & \ddots & \vdots & R^{-, 1} & R^{-, 0} & \ddots & \vdots \\
\vdots & \ddots & \ddots & R^{+, K-1} & \vdots & \ddots & \ddots & R^{-, K-1} \\
R^{+, K-1} & \cdots & R^{+, 1} & R^{+, 0} & R^{-, K-1} & \cdots & R^{-, 1} & R^{-, 0}
\end{array}\right)
$$




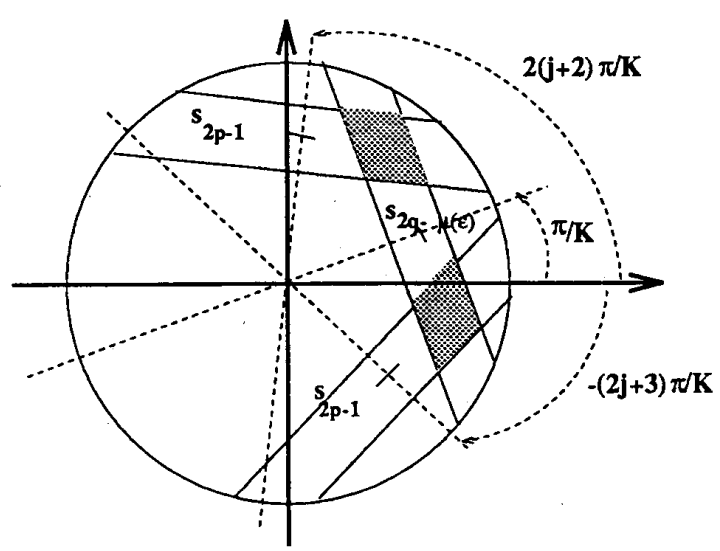

Fig. 6. $\left\langle l_{2(j+2), 2 p-1,}^{e} l_{1,2 q-\mu(\epsilon)}^{e}\right\rangle_{L^{2}(\Omega)}=\left\langle l_{2(K-1-j), 2 p-1}^{e}, l_{1,2 q-\mu(\epsilon)}^{e}\right.$ )$_{L^{2}(\Omega)}$

We summarize the results obtained in the next proposition Proposition 4: We have the following decomposition of the matrix:

$$
\begin{aligned}
& {\left[\begin{array}{ll}
\hat{R}^{+, k} & \hat{R}^{-, k}
\end{array}\right]} \\
& =U^{k}\left[\begin{array}{cccc}
\hat{R}^{+, k, 00} & \left(S^{k}\right)^{t} & \hat{R}^{-, k, 00} & S^{-, k, 01} \\
S^{k} & \hat{R}^{+, k, 11} & S^{-, k, 10} & \hat{R}^{-, k, 11}
\end{array}\right] V^{k}
\end{aligned}
$$

where

$$
U^{k}=\left[\begin{array}{cc}
e^{-i k \pi / 2 K} I_{\lceil P / 4\rceil} & 0 \\
0 & e^{i k \pi / 2 K} I_{\lceil P / 2\rceil-\lceil P / 4\rceil}
\end{array}\right]
$$

and the equation at the bottom of the page hold. $I_{n}$ is the identity matrix in dimension $n$. Thus, from (19) we see that the least squares problem (14) is reduced through discrete Fourier transforms of order $K$ and multiplications by roots of unity $e^{-i k \pi / 2 K}$ to the computation of the SVD of $K$ real rectangular blocks of dimension $\lceil P / 2\rceil \times 2\lceil P / 2\rceil$. Note that the computational cost is of the same order as for the standard scheme. Once the SVD is computed, the reconstruction cost is of the same order as for filtered backprojection. Thus, the DA is not only very interesting in the case of data scarcity, but also competitive in the case of fixed geometries with large $K$ (and $P$ ).

\section{NUMERICAL EXPERIMENTS}

In this section, we will show the efficiency of the proposed direct algebraic method. The new approach is compared to another algebraic method, the algebraic reconstruction technique (ART), which is extended to vector field tomography [21]. We show also that interlaced sampling schemes yield reconstructions almost as good as standard schemes when using the DA method. We propose to use a solenoidal testfield.

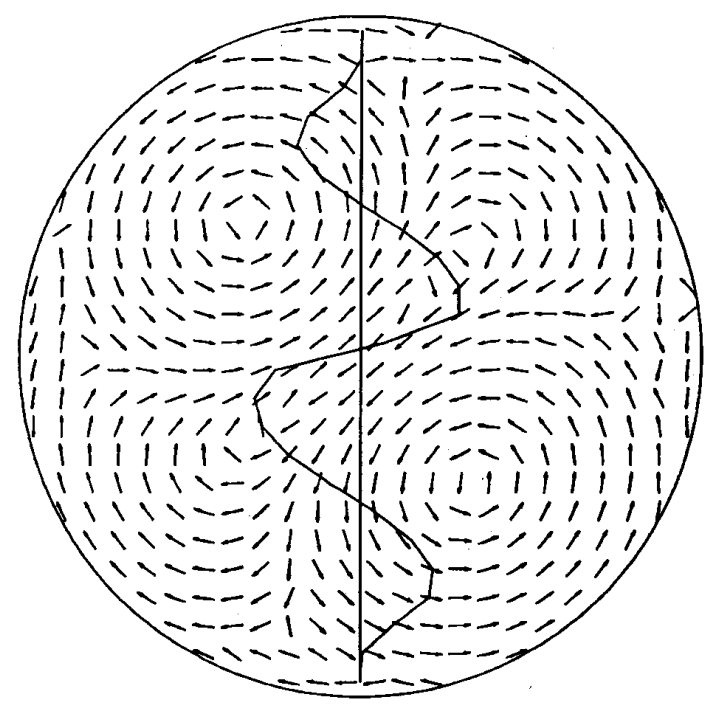

Fig. 7. Original testfield.

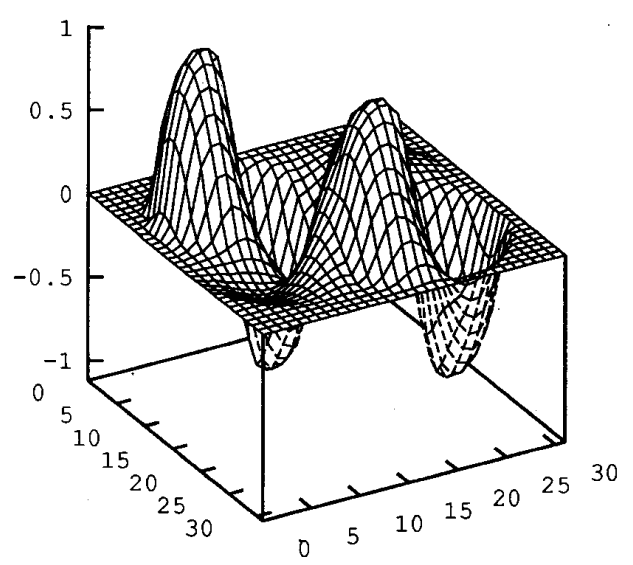

Fig. 8. Original $v_{1}$-component.

A plot of this field is given in Fig. 7. In this plot, all vectors have the same length to illustrate the orientation of the field. In Fig. 8, the component $v_{1}$ of the field is depicted as a 3d-plot. Since both fields are solenoidal, the longitudinal effect of (3) is used. As an error criterion we give the relative error

$$
E=\frac{\left\|\vec{v}_{\text {original }}-\vec{v}_{\text {reconst. }}\right\|}{\left\|\vec{v}_{\text {original }}\right\|} \cdot 100 \% \text {. }
$$

It is assumed that the field is sampled with $K=35$ views and $P=22$ rays per view. At first the field is reconstructed on a grid of $32 \times 32$ pixels with standard geometry. As it can be seen in Table I showing the results of the different

$$
V^{k}=\left[\begin{array}{cccc}
e^{i k \pi / 2 K} I_{[P / 4]} & 0 & 0 & 0 \\
0 & e^{-i k \pi / 2 K} I_{[P / 2\rceil-\lceil P / 4\rceil} & 0 & 0 \\
0 & 0 & e^{i k \pi / 2 K} I_{[P / 2\rceil-[P / 4]} & 0 \\
0 & 0 & 0 & e^{-i k \pi / 2 K} I_{[P / 4]}
\end{array}\right]
$$


TABLE I

Percentage of Relative Errors of the Simulations

\begin{tabular}{|r|c|c|c|c|c|}
\hline & \multicolumn{3}{|c|}{ D.A. } & \multicolumn{2}{c|}{ ART } \\
\cline { 2 - 6 } & $32 \times 32$ & $\begin{array}{c}32 \times 32 \\
\text { noisy data }\end{array}$ & $64 \times 64$ & $32 \times 32$ & $64 \times 64$ \\
\hline Standard & 9.85 & 16.3 & 10.3 & 22.8 & 43.8 \\
\hline Interlaced & 10.3 & 20.7 & 10.6 & - & - \\
\hline
\end{tabular}

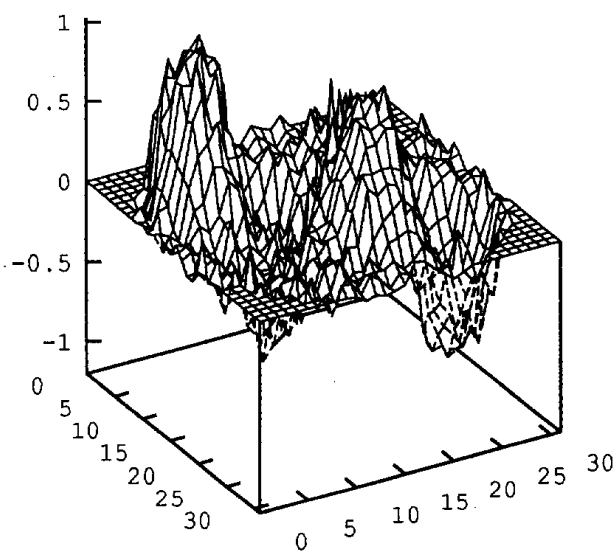

Fig. 9. Reconstruction with ART and standard scheme $(K=35, P=22$, i.e., $35 \times 22=770$ data).

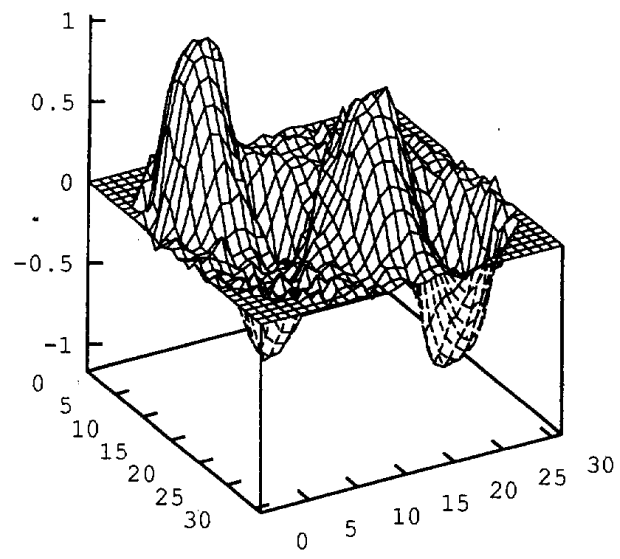

Fig. 10. Reconstruction with DA and standard scheme (770 data).

simulations, reconstructions with DA show fewer errors than reconstructions with ART.

Comparison of the plots of the reconstructed $v_{1}$-components in Figs. 9 and 10 elucidates that the reconstruction with ART is far more distorted by noise. Fig. 11 presents the $v_{1}$-component reconstructed with $\mathrm{DA}$ and interlaced data $(K=35, P=22$ : $35 \times 22 / 2$ data). Although only half of the data is used, almost no difference in the results obtained with the standard scheme can been seen. Indeed the relative error for the interlaced geometry is only slightly higher than for the standard type (see Table I). Reconstructions from interlaced data are, of course, more sensitive to noise; see Figs. 12 and 13. The advantage

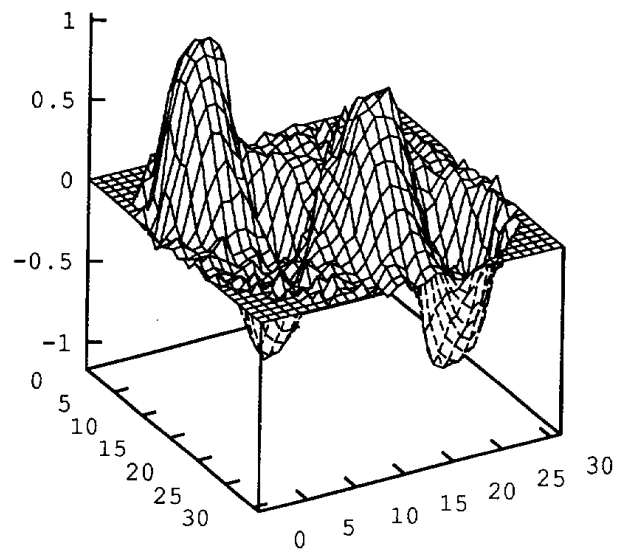

Fig. 11. Reconstruction with DA and interlaced scheme $(K=35, P=22$, i.e., $35 \times 22 / 2=385$ data).

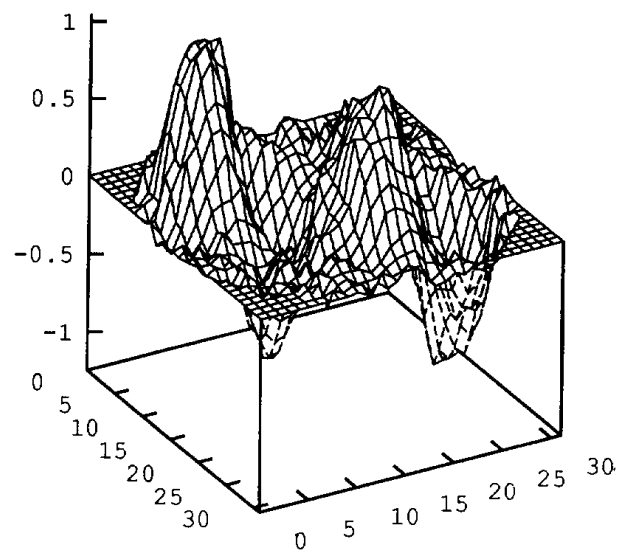

Fig. 12. Noisy data ( $\mathrm{SNR}=20$ ): Reconstruction with DA and standard scheme $(K=35, P=22)$.

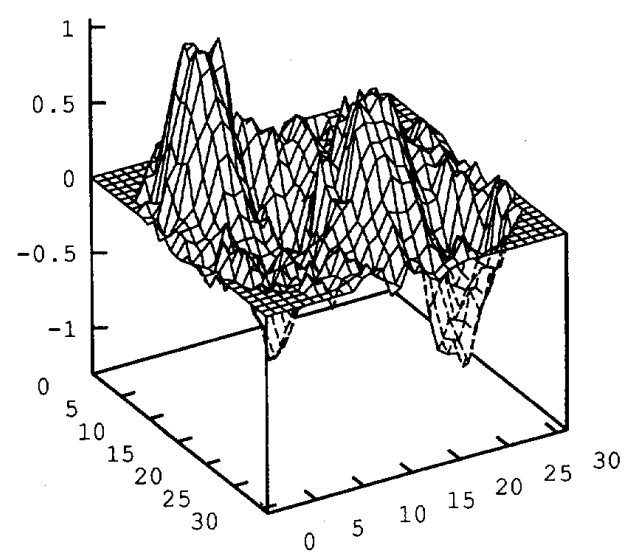

Fig. 13. Noisy data (SNR=20): Reconstruction with DA and interlaced scheme $(K=35, P=22)$.

of the new method becomes obvious if the image resolution is increased and the field is reconstructed on a grid of $64 \times 64$ pixels. In contrast with ART, which yields faulty results, 


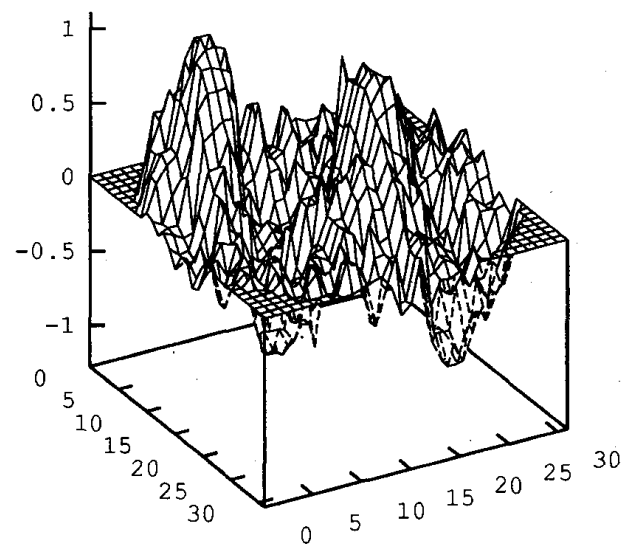

Fig. 14. Reconstruction with DA and standard scheme $(K=14, P=8)$.

with the DA approach, the same quality is realized for the reconstructions even if the data are reduced for the interlaced scheme. The DA solution does not depend on the number of pixels. The ART approach needs to be regularized when the number of pixels is too large. Enforcing the solution to be numerically $b$-bandlimited allows us to obtain good results even with interlaced data (see [6]).

If we have to make an estimate with only around 100 data, then interlaced data yield a more accurate reconstruction. In Fig. 14, we present the reconstruction of $v_{1}$ from the standard scheme $K=14, P=8$, or 112 data. In Fig. 16, we present the reconstruction of $v_{1}$ from the interlaced scheme $K=19, P=12$, or 114 data. In this case, the interlaced scheme yields a much better estimation of $v_{1}$ than a standard scheme having almost the same number of data. The interlaced reconstruction is in fact very close to the corresponding $19 \times 12$ standard reconstruction given in Fig. 15. These results are confirmed by the relative error values: $E=22.2$ for the $K=19, P=12$ standard scheme (with 228 data), $E=22.6$ for the $K=19, P=12$ interlaced scheme (with 114 data), whereas $E=36.9$ for the $K=14, P=8$ standard scheme (with 112 data).

\section{CONCLUSION}

We have proposed a new reconstruction method for vector field tomography. The approach is an adaptation of the DA method for scalar fields. It is shown that in the case of vector field tomography, the pleasant features of scalar tomography are preserved and a very efficient implementation of the algorithm is possible. We have also shown that the support of the Fourier transform of the projection of a vector field has the same specific bowtie-shaped form as in scalar tomography. This enables the use of an efficient interlaced sampling scheme which requires only half of the data needed for the standard sampling geometry to obtain the same quality of reconstruction. The proposed method is an adaptation of the DA algorithm used in the scalar case. We have proposed an efficient implementation of this method for interlaced data in vector field tomography that can be also used in the scalar case.

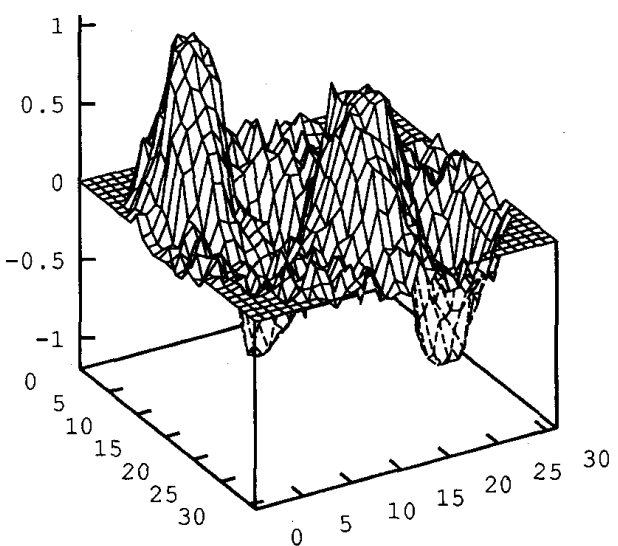

Fig. 15. Reconstruction with DA and interlaced scheme ( $K=19, P=12)$.

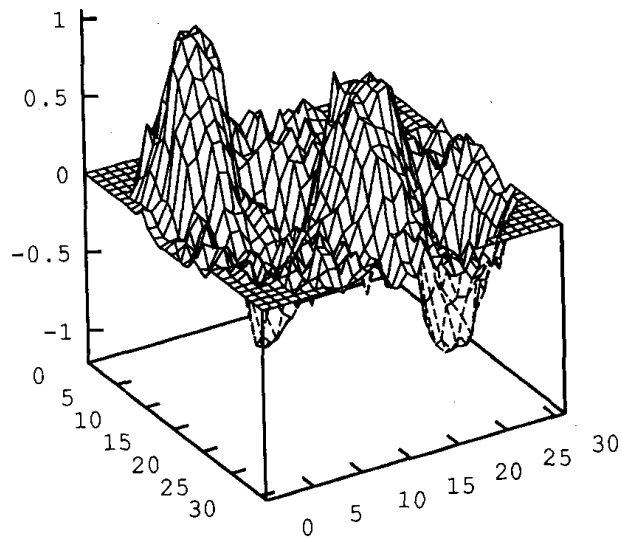

Fig. 16. Reconstruction with DA and standard scheme $(K=19, P=12)$.

These theoretical results were confirmed by a final simulation. With the proposed approach, a reconstruction method for vector fields is now available that yields reconstructions of high quality with half of the data needed for standard geometry and a minimum of computational cost.

\section{ACKNOWLEDGMENT}

The authors wish to thank K. Wright, English language consultant for LCI, for her help in proofreading and correcting this paper.

\section{REFERENCES}

[1] Bertero, De mol, and Pick, "Linear inverse problems with discrete data. I: General formulation and singular system analysis," Inverse Prob., vol. 1, pp. 301-330, 1985.

[2] H. S. Braun and A. Hauck, "Tomographic reconstruction of vector fields," IEEE Trans. Signal Processing, vol. 39, pp. 464-471, 1991.

[3] M. H. Buonocore, W. R. Brody, and A. Macovski, "A natural pixel decomposition for two-dimensional image reconstruction," IEEE Trans. Biomed. Eng., vol. BME-28, 69-78, 1981.

[4] A. M. Cormack, "Sampling the Radon transform with beam of finite width," Phys. Med. Biol., vol. 23, no. 6, pp. 1141-1148, 1978.

[5] L. Desbat, "Efficient sampling on coarse grids in tomography," Inverse Prob., vol. 9, pp. 251-269, 1993. 
[6] _ "Algebraic reconstructions from efficient sampling in tomography," in Proc. CT93, Novosibirsk, 1994.

[7] L. Desbat and P. Turlier, "Efficient reconstruction with few data in industrial tomography," in Proc. ECAPT92, 1992.

[8] A. Faridani, "An application of a multidimensional sampling theorem to computed tomography," in Proc. AMS-IMS-SIAM Conf. Integral Geom. Tomography, 1990, vol. 113, pp. 65-80.

[9] _ "A generalized sampling theorem for locally compact abelian groups," Math. Comp., vol. 63, no. 207, pp. 307-327, 1994.

[10] D. Girard, "Optimal regularized reconstruction in computerized tomography," SLAM J. Sci. Stat. Comput., vol. 8, no. 6, 1987.

[11] W. Klaverkamp, "Tomographische Bildrekonstruktion mit direkten algebraischen Verfahren," Ph.D. dissertation, Inst. f. Num. Math. Münster, 1991.

[12] F. Natterer, "Efficient implementation of optimal algorithms in computerized tomography," Math. Meth. in the Appl. Sci., vol. 8, no. 6, 1980.

[13] F. Natterer, The Mathematics of Computerized Tomography. New York: Wiley, 1986

[14] _ "Sampling in fan-beam tomography," SIAM J. Appl. Math., vol. 53, no. 2, pp. 358-380, 1993.

[15] S. J. Norton, "Tomographic reconstruction of 2-D vector fields: Application to flow imaging," Geoph. J., no. 97, pp. 161-168, 1988.

[16] _ "Unique tomographic reconstruction of vector fields using boundary data," IEEE Trans. Image Processing, vol. 1, pp. 406-412, 1992.

[17] D. P. Petersen and D. Middleton, "Sampling and reconstruction of wavenumber-limited functions in $N$-dimensional euclidean space," Inf. Contr., vol. 5, pp. 279-323, 1962.

[18] J. Radon, "Uber die Bestimmung von Funktionen durch ihre Integral durch ihre Integralwerte längs gewisser Mannigfaltigkeiten," Berichte Sächsische Akademie der Wissenschaften, vol. 29, pp. 262-279, 1917.

[19] P. A. Rattey and A. G. Lindgren, "Sampling the 2-D Radon transform," IEEE Trans. Acoust., Speech, Signal Processing, vol. ASSP-29, pp. 994-1002, 1981.

[20] T. Sato, H. Aoki, and O. Ikeda, "Introduction of mass conservation law to improve the tomographic estimation of velocity distribution from differential time-of-flight data," J. Acoust. Soc. Amer, vol. 6, pp. 2104-2106, 1985.

[21] B. Siemund, "Ein Beitrag zur computeromographischen Rekonstruktion von skalaren und vektorwertigen Funktionen," Ph.D. dissertation, Univ. of Duisburg, 1986.

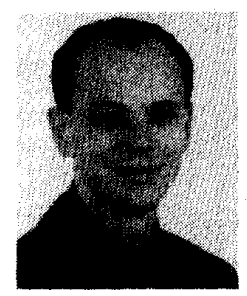

Laurent Desbat was born on October 2, 1964 in Lyon, France. He received the engineer degree of computer science and applied mathematics from the Polytechnical National Institute of Grenoble in 1987 and the Ph.D. degree in applied mathematics from the Joseph Fourier University of Grenoble in 1990.

After doing postdoctorate work in Münster, Germany, he joined the Technics of Image Processing, Modeling and Cognition Laboratory. He is now a full-time National Center of Scientific Research (CNRS) researcher. His research interests are inverse problems, tomography, efficient sampling, medical image processing, and parallel computing.

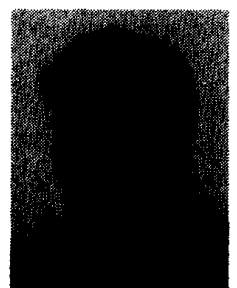

Andreas Wernsdörfer was born May 13, 1965 in Duisburg, Germany. He received the Masters degree in mechanical engineering from the University of Duisburg in July 1990 and the Ph.D. degree from the University of Karlsruhe in January of 1995.

Since September 1990, he has been a Scientific Assistant at the Department of Measurement and Control at the University of Karlsruhe. His main research interests are in vector field tomography and reconstruction from limited data. 